\title{
The paradox of the plankton: species competition and nutrient feedback sustain phytoplankton diversity
}

\author{
Kasia Kenitz ${ }^{1}$, Richard G. Williams ${ }^{1, *}$, Jonathan Sharples ${ }^{1,2}$, Özgür Selsil ${ }^{3}$, \\ Vadim N. Biktashev ${ }^{3}$ \\ ${ }^{1}$ School of Environmental Sciences, University of Liverpool, Liverpool L69 3GP, UK \\ ${ }^{2}$ National Oceanography Centre, Liverpool, Liverpool L3 5DA, UK \\ ${ }^{3}$ School of Mathematical Sciences, University of Liverpool, Liverpool L69 7ZL, UK
}

\begin{abstract}
The diversity of phytoplankton species and their relationship to nutrient resources are examined using a coupled phytoplankton and nutrient model for a well-mixed box. The phytoplankton community either reaches a competitive exclusion state, where there is an optimal competitor, or the abundance of each phytoplankton species continually varies in the form of repeating oscillations or irregular chaotic changes. Oscillatory and chaotic solutions make up over half of the model solutions based upon sets of 1000 separate model integrations spanning large, moderate or small random changes in the half-saturation coefficient, $K_{j i}$. The oscillatory or chaotic states allow a greater number of phytoplankton species to be sustained, even for their number to exceed the number of resources after additional species have been injected into the environment. The chaotic response, however, only occurs for particular model choices: when there is an explicit feedback between nutrient supply and ambient nutrient concentration, and when there are physiological differences among species, including cell quota and $K_{j i}$. In relation to the surface ocean, the nutrient feedback can be viewed as mimicking the diffusive nutrient supply from the nutricline. Inter-species competition might then be important in generating chaos when this diffusive transfer is important, but less likely to be significant when other transport processes sustain surface nutrient concentrations.
\end{abstract}

KEY WORDS: Plankton paradox $\cdot$ Coexistence $\cdot$ Chaos $\cdot$ Competitive exclusion $\cdot$ Phytoplankton communities

\section{INTRODUCTION}

Hutchinson (1961) first posed the paradox of the plankton: Why do so many phytoplankton species coexist while competing for a limited number of resources in a nearly homogeneous environment? For example, open ocean and lake surface waters usually contain the order of 1 to 10 dominant phytoplankton species together with many hundreds or more species at very low concentrations. This high number of phytoplankton species appears at odds with the competitive exclusion principle (Hardin 1960) where the

${ }^{*}$ Corresponding author. Email: ric@liverpool.ac.uk number of species coexisting at equilibrium is not expected to exceed the number of resources. For phytoplankton, the resources can be viewed in terms of macro nutrients, trace metals and variations in the light and temperature environment, such that if 2 phytoplankton species compete for the same resource, the most successful competitor is the one surviving on the minimum resource (Tilman 1977, Tilman et al. 1982). This excess in the number of phytoplankton species has been explained in terms of the phytoplankton system not reaching an equilibrium state due to temporal variability, as first specu-

() The authors 2013. Open Access under Creative Commons by Attribution Licence. Use, distribution and reproduction are unrestricted. Authors and original publication must be credited. 
lated in terms of seasonality by Hutchinson (1961) or spatial variability in the background environment (Richerson et al. 1970).

There are many ways in which this temporal and spatial variability can be achieved in the real world, such as from the externally imposed physical variability from changes in solar irradiance, weatherrelated changes in air-sea forcing and changes in mechanical forcing from tides. These changes in physical forcing can then shape the nutrient and light environment, and affect which phytoplankton species are likely to flourish. While this externally imposed variability is prevalent, there may also be internally induced cyclic behaviour allowing more species to be supported than the number of resources (Armstrong \& McGehee 1980). In particular, Huisman \& Weissing $(1999,2001)$ demonstrate how phytoplankton species consuming an abiotic resource can have a chaotic response; the phytoplankton abundance of each species does not reach an equilibrium, but instead continually evolves in a non-repeating sequence. Alongside this irregular behaviour, chaos is characterised by a high sensitivity to initial conditions; any differences in initial conditions exponentially increase in time and inhibit any predictability. With respect to the paradox of the phytoplankton, the number of phytoplankton species can exceed the number of resources in these chaotic solutions, subject to there also being a random injection of species into the environment (Huisman \& Weissing 1999; henceforth HW).

Here, we investigate the conditions for the phytoplankton community to exhibit chaotic, oscillatory and competitive exclusion solutions by addressing the dependence on the nutrient source and the fitness between species, as well as how long an intermittent addition of new species persists in the phytoplankton community.

\section{MODEL FORMULATION}

In this study, the coupled phytoplankton and nutrient model of HW is applied for a well-mixed box. The model is based on the linear chemostat assumption (Tilman 1977, 1980, Armstrong \& McGehee 1980, Huisman \& Weissing 1999), where there are $n$ phytoplankton species, $P_{i}$, competing for $k$ resources represented as nutrients, $N_{j}$

$$
\begin{array}{ll}
\frac{\mathrm{d} N_{j}}{\mathrm{~d} t}=D\left(S_{j}-N_{j}\right)-\sum_{i=1}^{n} Q_{j i} \gamma_{i}^{N} P_{i}, & j=1, \ldots, k \\
\frac{\mathrm{d} P_{i}}{\mathrm{~d} t}=P_{i}\left(\gamma_{i}^{N}-m_{i}\right), & i=1, \ldots, n
\end{array}
$$

$$
\gamma_{i}^{N}=\min \left(\frac{r_{i} N_{1}}{K_{1 i}+N_{1}}, \ldots, \frac{r_{i} N_{k}}{K_{k i}+N_{k}}\right)
$$

where the subscripts denote the particular species $i=$ $1, \ldots, \mathrm{n}$ and resources $j=1, \ldots, k$. In Eq. (1), the nutrient concentration, $N_{j,}$ evolves through a competition between a source from a nutrient supply and a sink from phytoplankton consumption: the nutrient supply involves an external supply, $S_{j,}$ and a feedback to $N_{j,}$ for each nutrient $j$, modulated by the system turnover rate, $D$, referred to as a dilution rate for a chemostat; the sink from the consumption by the sum of the phytoplankton species depends on the phytoplankton abundance, $P_{i}$, and growth rates, $\gamma_{i}^{N}$, for each species $i$ and the cell quota, $Q_{j i}$ for each species $i$ and nutrient $j$. In Eq. (2), each phytoplankton species, $P_{i}$ grows exponentially depending on the cell growth rate, $\gamma_{i}^{N}$, and mortality, $m_{i}$. In Eq. (3), the growth rate depends on the maximum growth rate, $r_{i r}$ for each species, modified by the abundance of the limiting nutrient relative to the half-saturation coefficient, $K_{j i}$, for each species and resource; note that for simplicity the growth rate does not depend on cell quota (as instead applied by Droop 1973). The chemostat model emulates steady state conditions where consumption of a resource is balanced by its import, and where maximum growth, resource requirements and external supply remain invariant in time.

We firstly consider cases with the same number of species and resources ( $n=k=5$; Figs. 1 to 5 ) and secondly where the number of species exceeds the number of resources ( $n>k=5$; Figs. 6 to 8 ). The model parameters and initial conditions follow those of HW unless otherwise stated (see Table S1 in the supplement at www.int-res.com/articles/suppl/m490p107_ supp.pdf).

We now examine the relationship between the abundance of phytoplankton species and nutrients, extending experiments by HW. The model solutions for the abundance of phytoplankton species reveal 3 different characteristic regimes: (1) competitive exclusion, when a long-term equilibrium is reached where one or more species dominate and drive the others to extinction (Fig. 1a); (2) repeating oscillations, when there is a repeating cycle in the abundance of each species (Fig. 1b); or (3) chaotic solutions, when there are non-repeating changes in species abundance (Fig. 1c). These differences start to become apparent over the first $100 \mathrm{~d}$ (Fig. 1, left panel). The character of the different responses is also reflected in the nutrient response in the wellmixed box: competitive exclusion leads to steady- 
(a) Competitive exclusion
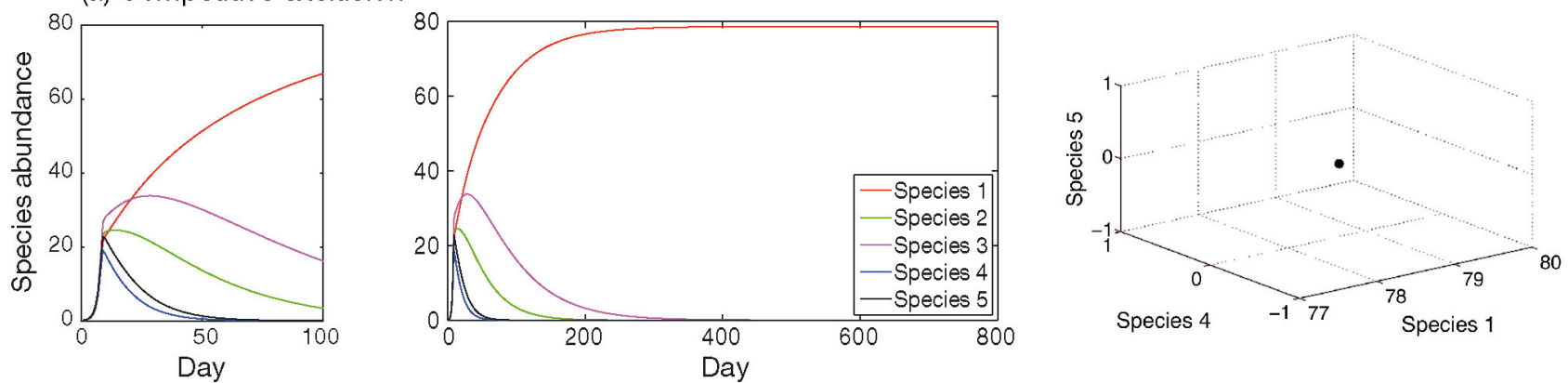

(b) Oscillatory response
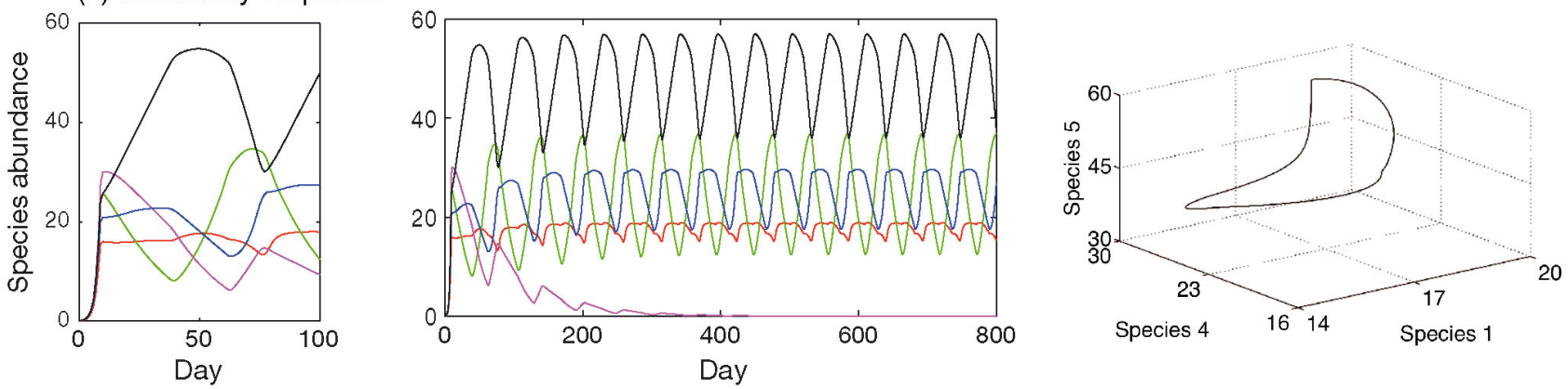

(c) Chaotic response
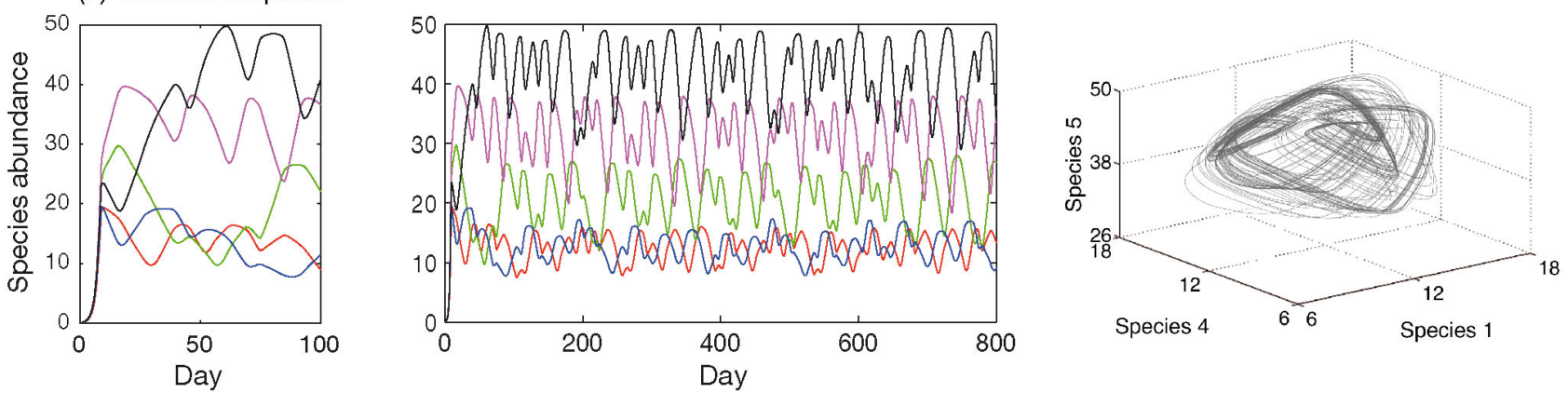

Fig. 1. Phytoplankton community response generated by the model of Huisman \& Weissing (1999) displaying sensitivity to the choice of the half-saturation coefficient. The model responses incorporate (a) competitive exclusion, (b) oscillations and (c) chaos, generated with $K_{4,1}=0.20, K_{4,1}=0.40$ and $K_{4,1}=0.30$ respectively, where $K$ is the half-saturation coefficient. The species responses are shown for the initial period of $100 \mathrm{~d}$ and over $1000 \mathrm{~d}$ (left and central panels), and their phase diagrams are from 500 to $5000 \mathrm{~d}$ (right panels)

state nutrient concentrations sustained by their nutrient source, while oscillations or chaos within the phytoplankton community are associated with periodic or irregular fluctuations in the ambient nutrient concentrations.

In terms of the 'paradox of the phytoplankton', both the repeating oscillations and chaotic solutions are of interest as a long-term equilibrium is not reached, part of the explanation suggested by Hutchinson (1961). Taking that view further forward, HW argued that a chaotic state enables the number of species to exceed the number of resources.

In our model diagnostics, whether chaos is obtained is formally identified using the following approaches. Firstly, the temporal changes in phyto- plankton abundance are illustrated by a trajectory in a phase space, where each dimension represents the abundance of a particular phytoplankton species. For example, consider the evolution of 3 arbitrary species in a 3-D phase diagram (Fig. 1, right panels): competitive exclusion is represented by a single point; repeating oscillations by repeating closed trajectories; and chaotic solutions by irregular and continually changing trajectories. Secondly, the sensitivity to initial conditions can be estimated by evaluating the rate at which 2 points in phase space, initially close together, subsequently diverge away from each other. This diagnostic, referred to as the maximal Lyapunov Exponent (Kantz 1994), is often used to define chaos, identifying when there is an exponential increase in 
the separation of 2 trajectories. Thirdly, we employ a binary test distinguishing chaos from non-chaotic dynamics, referred to as the 0-1 test for chaos, adjusted to detect weak chaos (Gottwald \& Melbourne 2004, 2009). This technique is the most efficient approach when there are many repeated model integrations. Further explanation of these methods is provided in the Supplement.

\section{MODEL SENSITIVITY EXPERIMENTS}

Sensitivity experiments are now performed to understand the different ecosystem response in the well-mixed box, focussing in turn on the environmental control via the nutrient supply, the physiological control of each species via the cell quota and $K_{j i}$ coefficient, and the effect of random injections of different phytoplankton species.

\section{Environmental control by nutrient supply}

The nutrient supply in Eq. (1) includes an external supply, $D S_{j}$, and a feedback term, $-D N_{j}$, to ambient nutrient concentrations. The external supply and feedback together act to restore nutrient concentrations, which can be viewed as a crude way of replicating how physical processes act to supply nutrients and sustain biological productivity. For example, in a vertical water column, phytoplankton consume inorganic nutrients in the euphotic zone, and these inorganic nutrients can be resupplied by vertical diffusion, acting to transfer nutrients down gradient from high concentrations in the nutricline to the surface. This diffusive nutrient supply is given by $\frac{\partial}{\partial z}\left(\kappa \frac{\partial N}{\partial z}\right)$, which, applying scale analysis, is typically $-\frac{\kappa}{\Delta z^{2}}\left(N_{\text {surface }}-N_{\text {nutricline }}\right)$, where $\kappa$ is the vertical diffusivity, $N_{\text {surface }}$ and $N_{\text {nutricline }}$ are the nutrient concentrations at the surface and nutricline, separated by a vertical spacing $\Delta z$. Thus, when $N_{\text {surface }}<N_{\text {nutricline, }}$ diffusion acts to restore the surface nutrients towards the value in the nutricline, reducing the contrast between $N_{\text {surface }}$ and $N_{\text {nutricline, so acting in a similar }}$ manner to $-D N_{j}$ in the nutrient supply in Eq. (1).

To assess the effect of the nutrient feedback in Eq. (1), model experiments are performed with the nutrient supply taking the form $D\left(S_{j}-\alpha N_{j}\right)$ where $\alpha$ ranges from 0 to 1 (and otherwise default model parameters are used; Table S1 in the Supplement). The factor $\alpha$ controls the net amount of nutrient supplied into the environment, and measures the strength of feedback to the nutrient resource. At weak to moder- ate feedback $(\alpha<1)$, there are repeating cycles of a single species dominating, switching later to a different single species, and this pattern is progressively repeated (Fig. 2a). Increasing the feedback leads to a reduction in the period of each cycle (Fig. 2a,b).

For strong feedback $(\alpha \sim 1)$, there are always timevarying changes in the abundances of the 5 species and a chaotic response, when the sequences for the abundances of phytoplankton species do not exactly repeat in time (Fig. 2c), as evident in their trajectories not repeating in phase diagrams. Thus, the presence of $-D N_{j}$ in Eq. (1) fundamentally affects the nature of the phytoplankton solutions.

While some form of nutrient feedback is plausible given how diffusion acts to supply nutrients to the surface, other physical transport processes often dominate over this diffusive supply, such as entrainment at the base of the mixed layer, and the horizontal and vertical transport of nutrients (Williams \& Follows 2003). Hence, the nutrient feedback acting to restore surface nutrients is unlikely to hold all the time, possibly varying in an episodic manner, and probably depending on the physical forcing and background circulation. Accordingly, we now consider the effect of introducing slight modifications in $-D N_{j}$ in model experiments using the default chaotic parameters.

(1) The nutrient supply, $D\left(S_{j}-N_{j}(t)\right)$, is now interspersed by intermittent periods when there is no feedback, such that the supply temporarily increases to $D S_{j}$ for short periods ranging from $10 \mathrm{~min}$ to $8 \mathrm{wk}$ (Fig. 3a, shaded). During the intermissions, the phytoplankton solutions move towards a single species dominating at any single time (Fig. 3a, upper panel), rather than 5 species being sustained; this response is more apparent for prolonged periods without relaxation. After the intermissions, the nutrient supply returns to including the nutrient feedback, and the phytoplankton solutions return to being chaotic (Fig. 3a). In terms of the nutrient forcing, the nutrient sources for this case with intermissions and the default case without intermissions (Fig. 1c) are initially identical, but then differ after the first intermission due to the different evolution of the nutrients (Fig. 3a, lower panel).

(2) The model solutions are altered if the nutrient supply is adjusted to $D\left(S_{j}-\tilde{N}_{j}\right)$, where $\tilde{N}_{j}$ represents the past record of forcing based upon the default $N_{j}(t)$ record (shown to trigger the chaotic response in Fig. 1c with $\alpha=1$ ), but now including prescribed intermissions. After the first intermission, the lack of any interactive nutrient feedback leads to the phytoplankton solutions changing from being chaotic and 
evolving to a single species dominating (Fig. 3b); the dominant species can alternate in time with a period lengthening with every cycle, referred to as heteroclinic cycles (Huisman \& Weissing 2001). The nutrient source in this case and the default are nearly identical (Fig. 3b, lower panel), but the lack of any interactive adjustment prevents the chaotic solutions being sustained. Thus, the presence of the interactive feedback is crucial for the chaotic solutions to emerge and persist.

(3) Given the importance of the nutrient feedback, the effect of a slight delay is now introduced into the nutrient supply (an arbitrary lag of 1 day), so that the supply term becomes $D\left(S_{j}-N_{j}(t-1\right.$ day $\left.)\right)$. The nutrient supply retains the interactive feedback, although the lag implies that the nutrient supply is not exactly the same as in the chaotic case (1) (Fig. 3a). However, including the temporal lag does not significantly alter the character of the solutions: chaos is either sustained or moves to multiple-period oscillations (Fig. 3c) with all 5 species persisting and varying in time.

In summary, the chaotic nature for the abundance of the phytoplankton species is reliant on there being a feedback to the nutrient concentration: an absent or too weak feedback leads to competitive exclusion or oscillatory changes in the dominant phytoplankton species, which sustain fewer species at any particular time. In partial accord with this viewpoint, chemostat laboratory experiments find that the community response is sensitive to nutrient supply rates (Becks et al. 2005), where the nutrient supply is modelled with feedback terms as in Eq. (1).
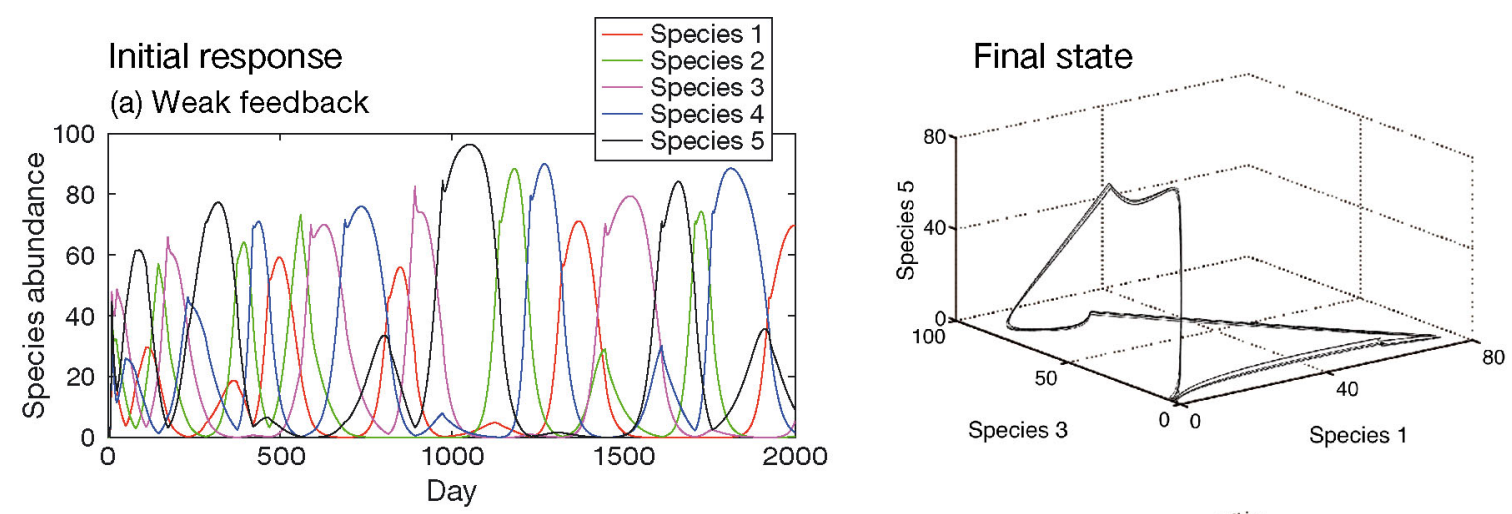

(b) Moderate feedback
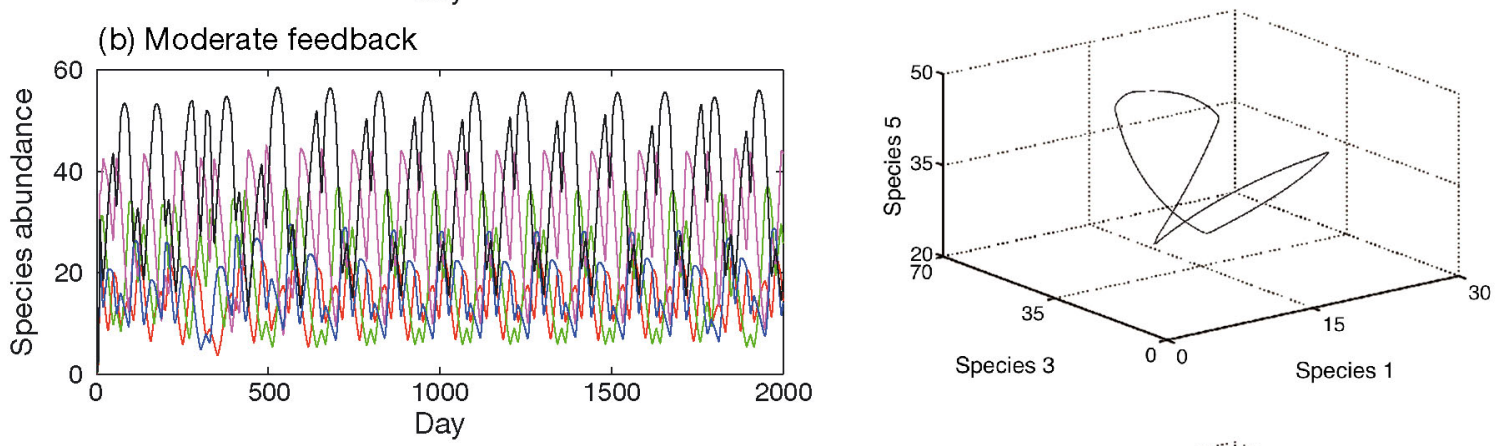

(c) Strong feedback
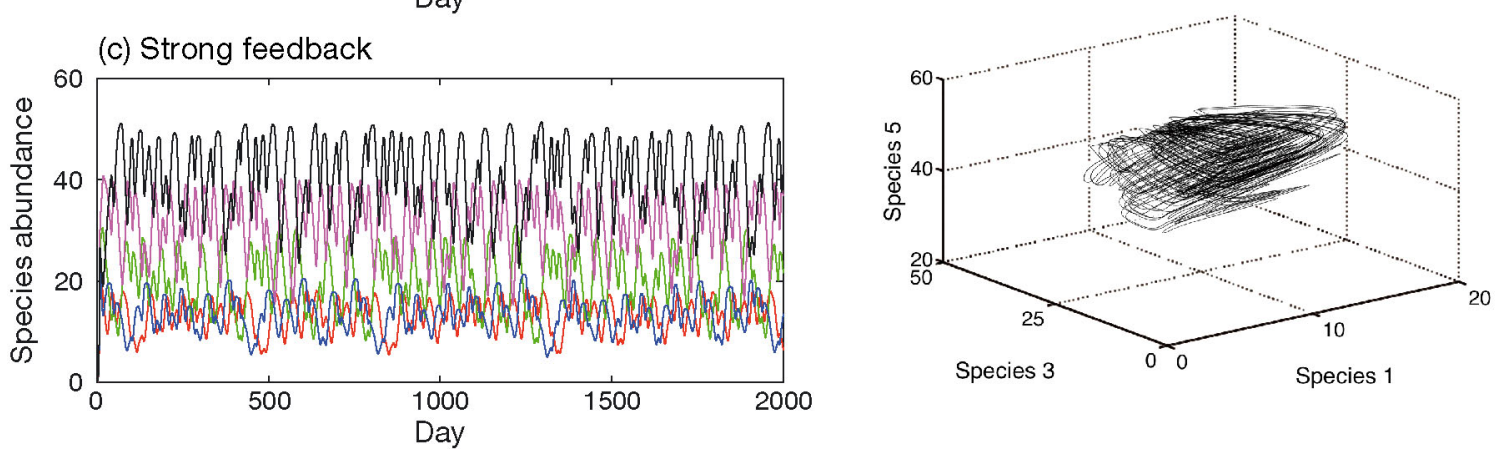

Fig. 2. Phytoplankton community response to the nutrient supply, $D\left(S_{j}-\alpha N_{j}\right)$ (for abbreviations see 'Model formulation'), with varying levels of feedback: (a) weak $(\alpha=0.2)$, (b) moderate $(\alpha=0.6)$, and (c) strong $(\alpha=0.8)$. Time series plots are for the initial $2000 \mathrm{~d}$ (left panels) and phase plots over the later 7000 to $10000 \mathrm{~d}$ (right panels) 
(a) Feedback with breaks
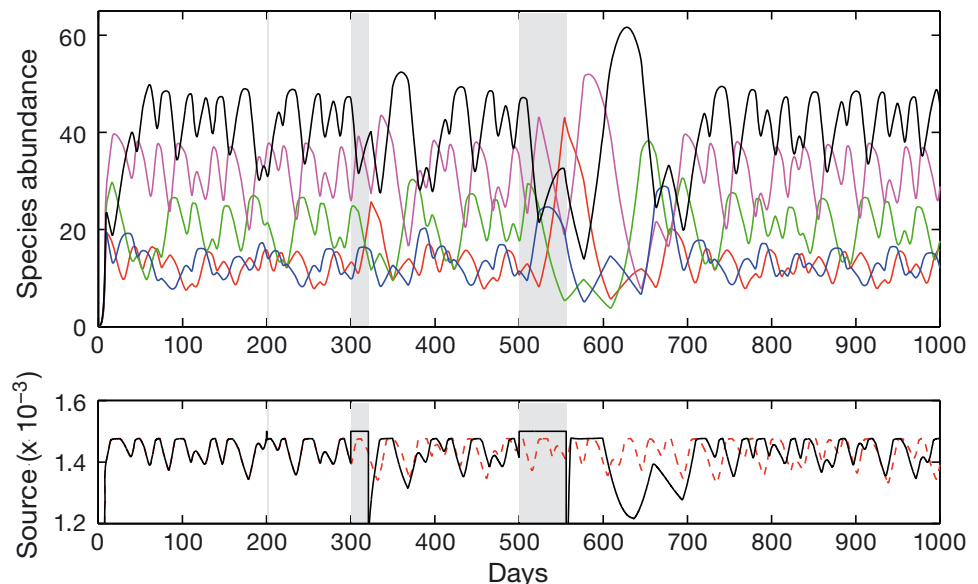

(b) No interactive feedback
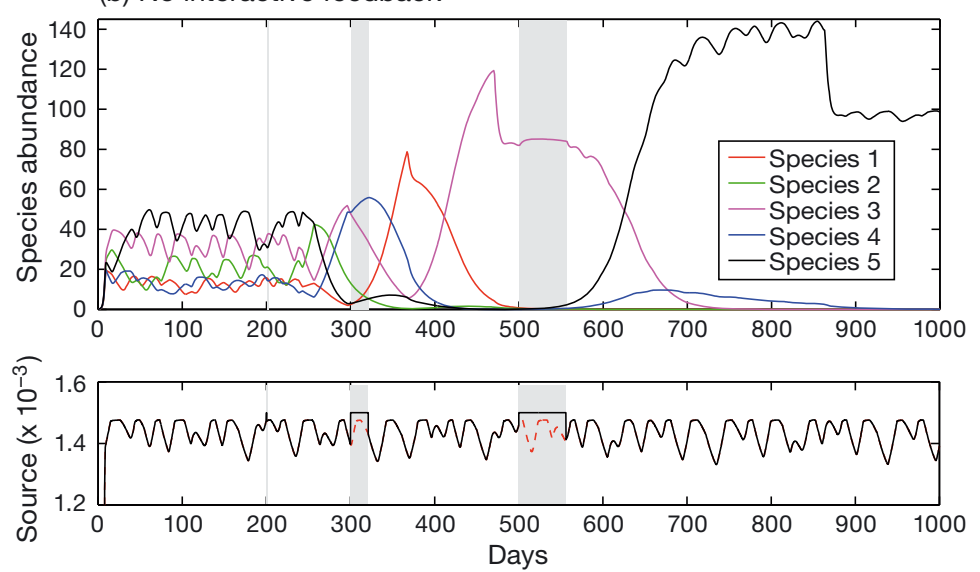

(c) Lagged feedback
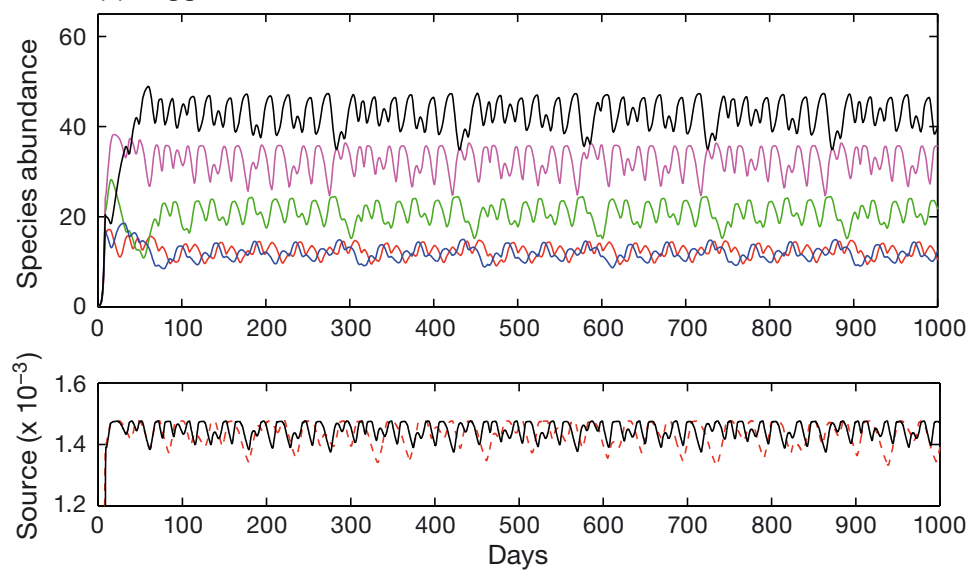

Fig. 3. Phytoplankton species abundance (upper panels) and nutrient source (lower panels) versus time with the modified nutrient supply: (a) nutrient source with feedback and intermittent disruptions (grey shading) lasting 10 min (Day 200), 3 wk (Day 300) and 8 wk (Day 500), when the default nutrient feedback is temporarily removed, $D S_{j i}$ (b) nutrient source without feedback defined by the record of the default nutrient source (as in Fig. 1c) including intermissions (as in Panel a); (c) nutrient source with lagged feedback, where nutrient supply depends on the nutrient concentration from the previous day, $D\left(S_{j}-N_{j}(t-1\right.$ day $\left.)\right)$. In each case, the time series of the nutrient source for resource 1 (black line) is compared with that for the default source term (dashed red line) in the bottom panels. For abbreviations see 'Model formulation'

\section{Physiological choices}

Physiological traits and related trade-offs define the ecological niche of species and affect their survival ability. The effect of modifying the choice of cell quota and $K_{j i}$ is now assessed on the phytoplankton community structure.

\section{Cell quota}

In a similar manner to the way the nutrient relaxation is investigated, the cell quota, $Q_{j i}$ is assumed either (1) to be the same for all species and alter in the same manner for each resource, or (2) to vary in a different manner for each species and resource (following HW):

$$
\begin{aligned}
Q_{j i} & =\left(\begin{array}{ccccc}
0.04 & 0.04 & 0.04 & 0.04 & 0.04 \\
0.08 & 0.08 & 0.08 & 0.08 & 0.08 \\
0.10 & 0.10 & 0.10 & 0.10 & 0.10 \\
0.03 & 0.03 & 0.03 & 0.03 & 0.03 \\
0.07 & 0.07 & 0.07 & 0.07 & 0.07
\end{array}\right)+ \\
& \beta\left(\begin{array}{ccccc}
0.0 & 0.0 & 0.03 & 0.0 & 0.0 \\
0.0 & 0.0 & 0.0 & 0.02 & 0.0 \\
0.0 & 0.0 & 0.0 & 0.0 & 0.04 \\
0.02 & 0.0 & 0.0 & 0.0 & 0.0 \\
0.0 & 0.02 & 0.0 & 0.0 & 0.0
\end{array}\right)
\end{aligned}
$$

where the values in the matrix for $Q_{j i}$ are for each resource $j$ in the rows and for each species $i$ in the columns, and $\beta$ varies from 0 to 1 ; other model parameters are the default (Table S1). A choice of $\beta$ to 0 represents the same cell quota for all species, while $\beta$ to 1 is representative of HW with an increase in the contrast in cell quota for a particular resource for each species. When the cell quota is identical for each species, there is competitive exclusion (Fig. 4a) and the fittest species has the lowest requirement for the limiting resource (Tilman 1977). When moderate changes in cell quota are chosen, there are oscillations in the phytoplankton response (Fig. 4b). When large contrasts in cell quota are chosen for each species, there are chaotic fluctuations in the concentrations of each phytoplankton species (Fig. 4c), allowing the coexistence of all 5 species. 
(a) Nearly uniform cell quota $Q_{j i}$
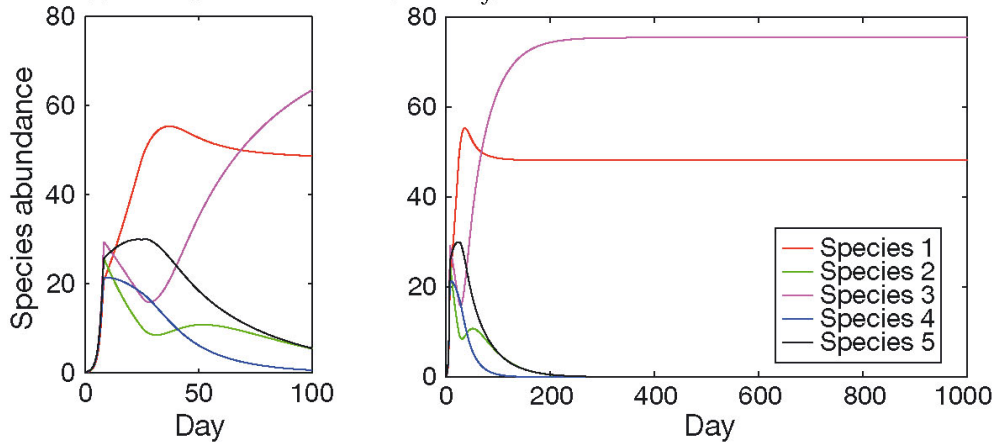

(b) Moderate changes in cell quota $Q_{j i}$
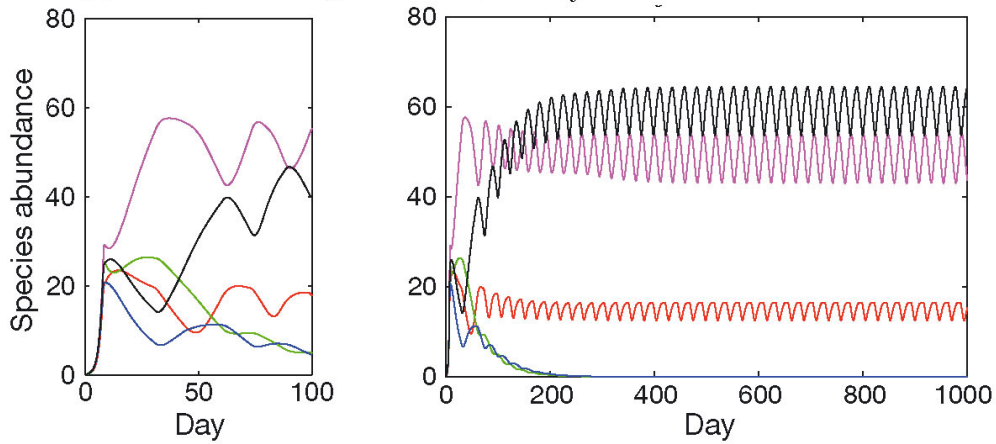

(c) Large changes in cell quota $Q_{j i}$
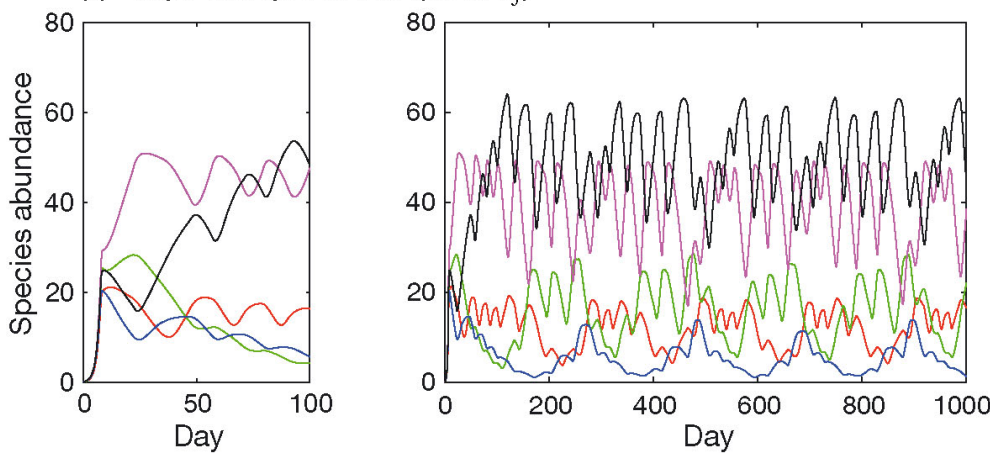
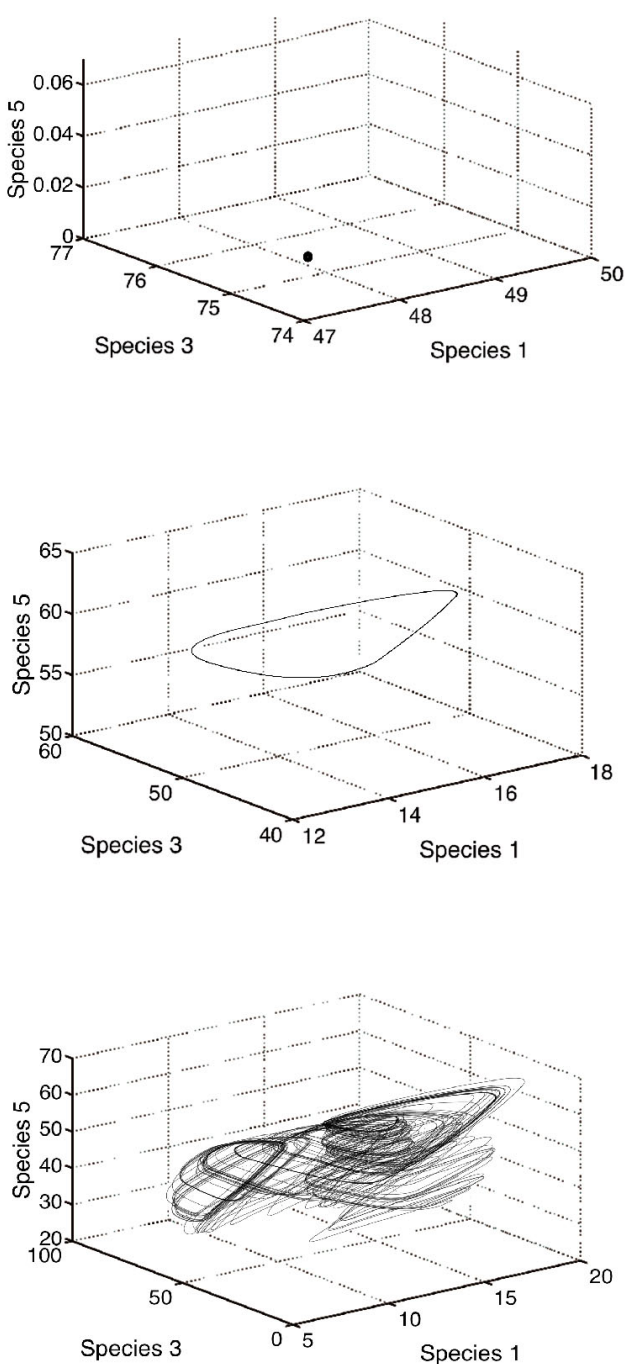

Fig. 4. Phytoplankton community response to changes in cell quota, $Q_{j i}$ : (a) nearly uniform cell quota for each species, $\beta=0.2$; (b) moderate contrasts in cell quota for each species, $\beta=0.6$; and (c) strong contrasts in cell quota, $\beta=0.7$. The temporal adjustment is shown over the first 100 and $1000 \mathrm{~d}$ (left and middle panels) and corresponding phase plots (right panels) for abundance of species 1,3 and 5 for 500 to $5000 \mathrm{~d}$

\section{Half-saturation coefficient}

The sensitivity to the half-saturation coefficient, $K_{j i}$, is investigated by varying the values for each species and resource, but in an ordered manner so that each of the species is the optimal competitor for one of the resources:

$$
K_{j i}=\left(\begin{array}{lllll}
k_{5} & k_{4} & k_{3} & k_{2} & k_{1} \\
k_{1} & k_{5} & k_{4} & k_{3} & k_{2} \\
k_{2} & k_{1} & k_{5} & k_{4} & k_{3} \\
k_{3} & k_{2} & k_{1} & k_{5} & k_{4} \\
k_{4} & k_{3} & k_{2} & k_{1} & k_{5}
\end{array}\right)
$$


dominant species (Fig. 5, blue), oscillations with a repeating cycle in species abundance or irregular chaos, both involving all 5 species (Fig. 5, green and red respectively). For competitive exclusion, the dominant species might alter and be replaced by another species, taking the form of heteroclinic cycles (as shown earlier in Fig. 3b); the resulting ordered sequence is a consequence of each species being the optimal competitor for a different resource.

A pattern in the different model responses is evident when comparing the competitive ability of the intermediate species with the other competitors (Fig. 5). For the intermediate competitor, $k_{3}$, compared with the 2 strongest competitors, $k_{1}$ and $k_{2}$, competitive exclusion is the most likely response when species are of comparable fitness, but alters to chaos and then oscillations with greater contrasts in the strength of these competitors (Fig. 5, left panels). Hence, the more competitive the intermediate competitor, the more chance of there being an optimal competitor and obtaining competitive exclusion, while a weaker intermediate competitor encourages chaos or oscillations.

(a) Large changes in half-saturation coefficient, $K_{j i}$
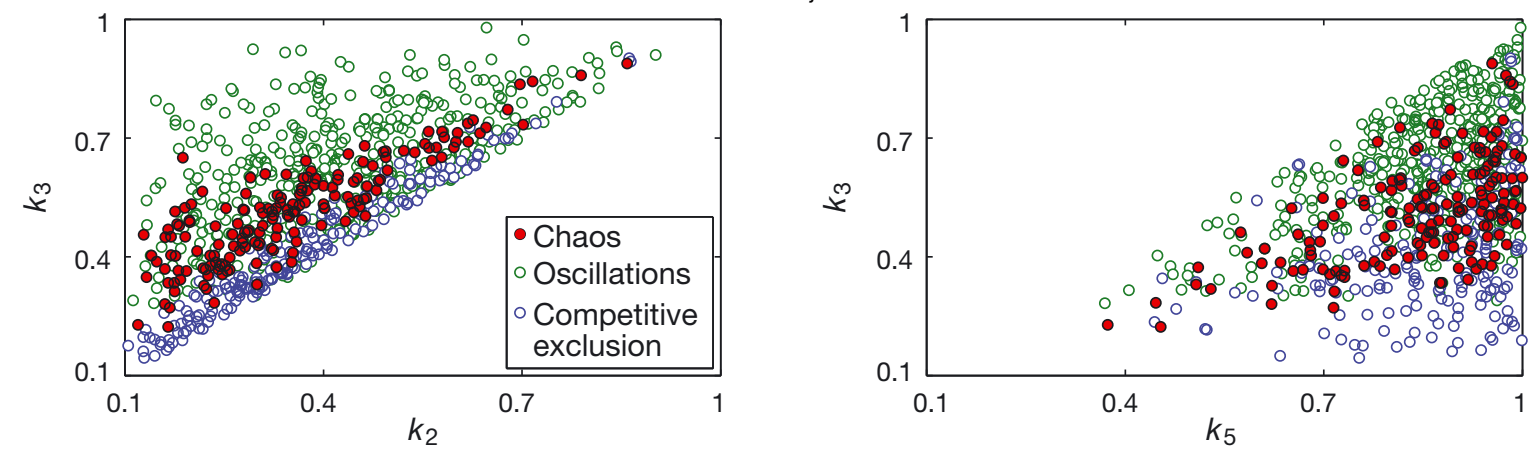

(b) Moderate changes in half-saturation coefficient, $K_{j i}$
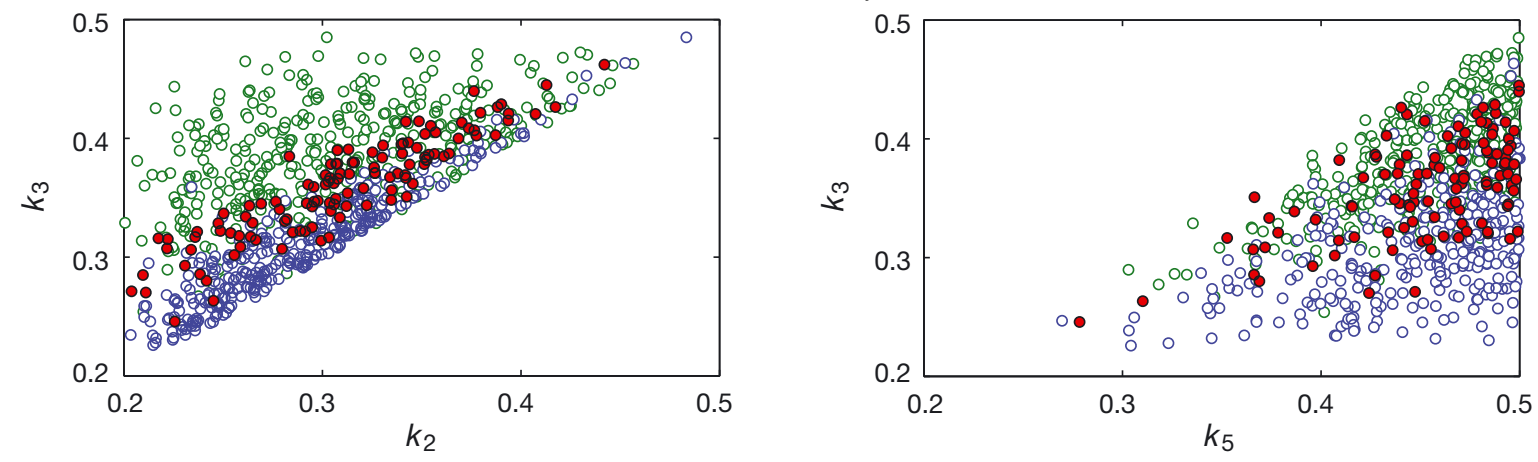

(c) Small changes in half-saturation coefficient, $K_{j i}$
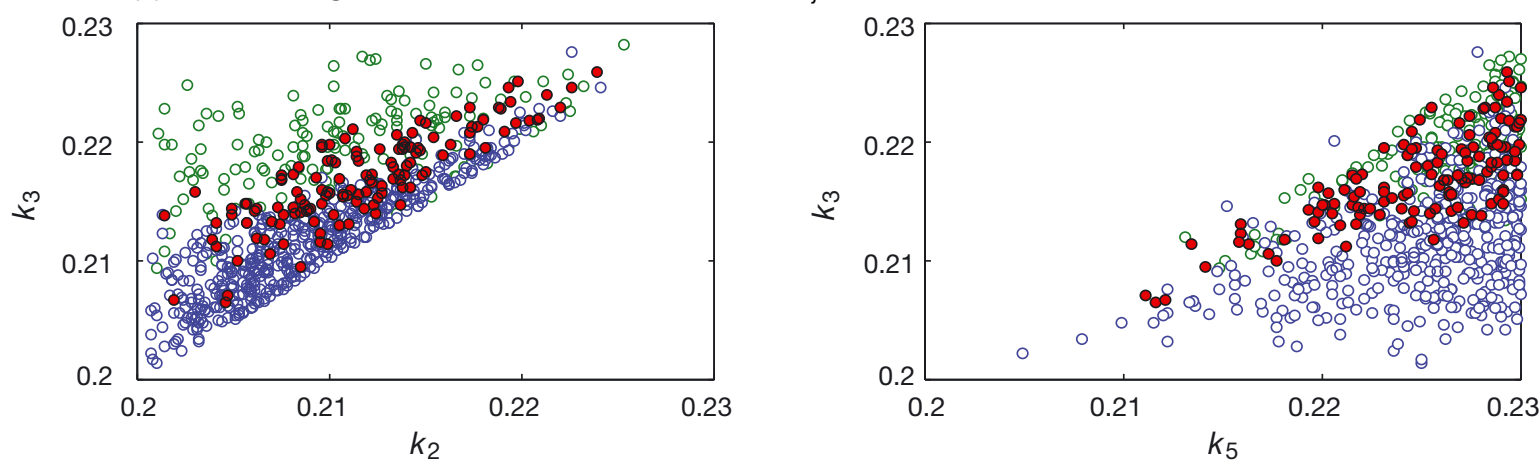

Fig. 5. The phytoplankton community response to randomly assigned half-saturation coefficient, $K_{j i}$, within prescribed bounds for 1000 model integrations, each lasting $50000 \mathrm{~d}$. The model responses include competitive exclusion with 1 dominant species at any time (blue), oscillations (green) and chaos (red). Illustrated are the relationships between different $K_{j i}$ for (a) large, (b) moderate and (c) small contrasts. The model solutions for a strong versus intermediate competitor, $k_{2}$ versus $k_{3}$ are shown in the left panels, and a weak versus intermediate competitor, $k_{5}$ versus $k_{3}$ in the right panels 
The other side of this response is that comparing the intermediate competitor, $k_{3}$, with the 2 weakest competitors, $k_{4}$ and $k_{5}$, leads to the reversed pattern (Fig. 5, right panels): a similar fitness of the 3 species favours oscillations, while increased contrasts generally lead to chaos and eventually are more likely to lead to competitive exclusion. Indeed, the more similar the intermediate competitor is to the weaker species, the more the intermediate competitor differs from the strong competitors, which explains the reversed pattern. No regular structure is evident when $K_{j i}$ are compared for strong versus weak competitors.

When the perturbations in $K_{j i}$ are in a very narrow range (0.2 to 0.23$)$, competitive exclusion is the dominant response, occurring over $47 \%$ of the parameter space, while oscillations occur in $17 \%$ and chaotic solutions in $14 \%$ of parameter space (Table 1 ); the remaining $22 \%$ of solutions are not distinguished between oscillations and chaos. When the perturbations in $K_{j i}$ are in a larger range (0.1 to 1.0 ), competitive exclusion reduces to $19 \%$ of parameter space and instead oscillations increase to $45 \%$ and chaos to $17 \%$ of parameter space. Hence, when $K_{j i}$ of intermediate and strong competitors are close together, there is more chance of identifying the optimal competitor and obtaining competitive exclusion.

\section{Random injection of phytoplankton species}

We next investigate the response of the model to an intermittent 'injection' of new species, replicating how ocean circulation leads to the transport and dispersal of phytoplankton species.

To investigate this species injection and the longerterm community response, an 'invasion approach' is applied broadly following Huisman et al. (2001): additional species are introduced with 3 new species with initial abundance $P_{i}=0.1$, typically introduced every $30 \mathrm{~d}$ (with random deviations of a maximum of

Table 1. Different phytoplankton community responses (\%) for 3 separate sets of 1000 model integrations, each with a different range of randomly generated half-saturation coefficient, $K_{j i}$. For a proportion of model simulations, community behaviour could not be distinguished between oscillations and chaos

\begin{tabular}{|lcccc|}
\hline$K_{j i}$ range & $\begin{array}{c}\text { Competitive } \\
\text { exclusion }\end{array}$ & $\begin{array}{c}\text { Oscilla- } \\
\text { tions }\end{array}$ & Chaos & $\begin{array}{c}\text { Oscillations } \\
\text { or chaos }\end{array}$ \\
\hline $0.2-0.23$ & 47 & 17 & 14 & 22 \\
$0.2-0.5$ & 32 & 40 & 12 & 16 \\
$0.1-1.0$ & 19 & 45 & 17 & 19 \\
\hline
\end{tabular}

10 d), starting at Day 90 and persisting for 1 yr. The additional species have their cell traits stochastically determined for each model integration, with $K_{j i}$ chosen within the interval 0.2 to 0.5 , and $Q_{j i}$ within the interval 0.01 to 0.1 . These biological parameters were assigned for each species and resource either in a random manner, or assuming a negative relation between fitness and cell quota (scenarios 1 and 3 of Huisman et al. 2001, respectively); however, the longterm character of the model results turned out not to be sensitive to these scenarios.

The model state prior to the invasion is our default choice: 5 species competing for 5 resources, so that competition theory predicts that up to 5 different species should be sustained for a long-term equilibrium. To sample the different characteristic regimes, the model experiments are repeated for a range of choices for $K_{j i}$ : obtaining (1) chaos with the default $K_{j i}$ matrix, (2) single-period oscillations with $K_{5,4}=0.37$, and (3) competitive exclusion with $K_{2,4}=0.20$; with otherwise default choices for the rest of $K_{j i}$ for all 3 cases.

In the chaotic case, the number of phytoplankton species exceeds the number of resources over the length of the integration of $2500 \mathrm{~d}$ (Fig. 6a, panel [i]). Chaotic fluctuations then allow the number of species to exceed the number of resources, referred to as 'supersaturation', in our integrations supporting 20 to 30 species within 3 months from the last input of new species (Fig. 7a). The number of coexisting species gradually reduces to 10-15 surviving species after $1 \mathrm{yr}$, and decreases further to less than 5 after 2 yr for the majority of the model compilations. The chaotic fluctuations can sometimes abruptly diminish (Fig. 6a, panel [ii]), without any intermittent disruption prior to the event. Thus, the fittest competitors persist, while the weaker species progressively become extinct. During the process of introducing more species, there is more chance for an optimal competitor to be identified and so there is less chance for chaos and oscillations to emerge.

Oscillatory solutions lead to a broadly similar response to chaotic solutions: there is a supersaturation in the number of species, which gradually declines in time, as illustrated for 1-period oscillations (Figs. 6b $\& 7 \mathrm{~b}$ ) and also obtained for 2-period oscillations (not shown).

In the case of competitive exclusion, the community is already dominated by an optimal competitor and so there is a very weak, short-lived response to an injection of additional species (Fig. 6c). Supersaturation is only sustained for a brief 6-month period after the last input of additional species, swiftly returning to fewer than 5 coexisting species (Fig. 7c). 
(a) Chaos

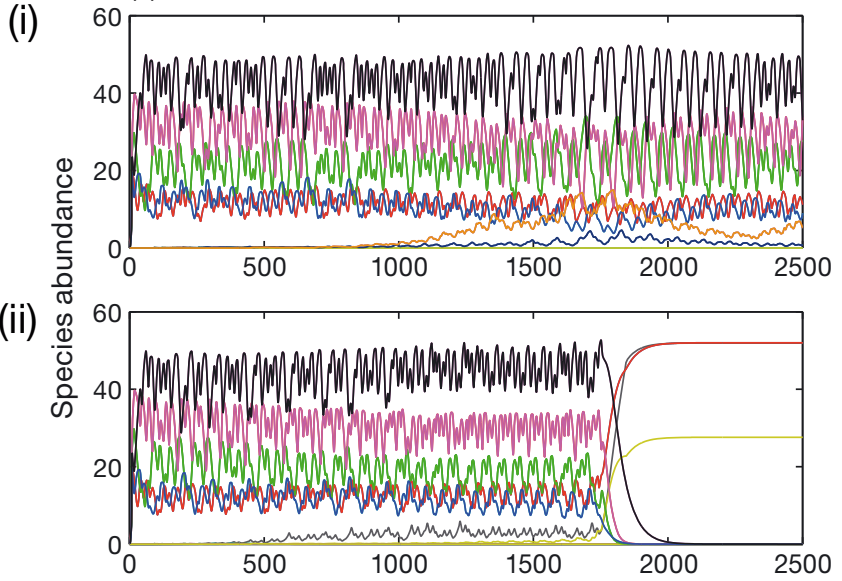

(b) Oscillations

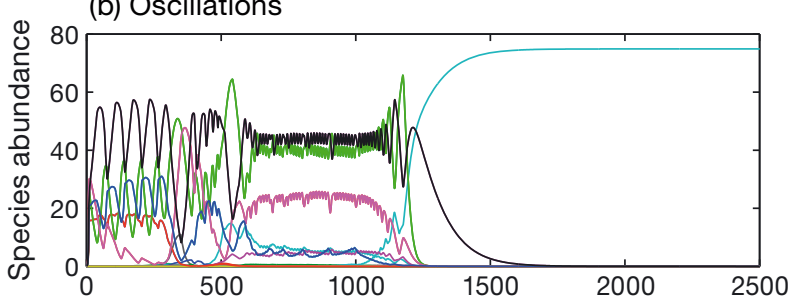

(c) Competitive exclusion

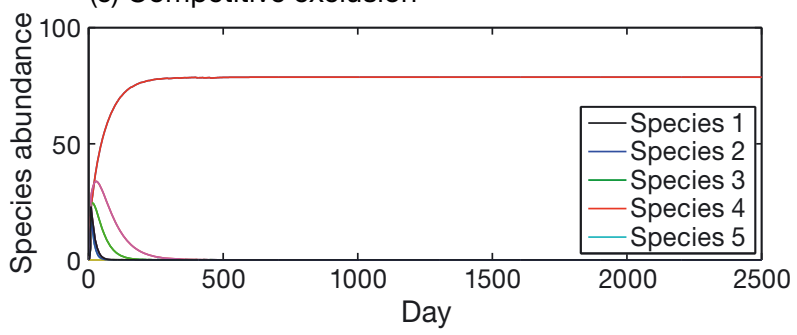

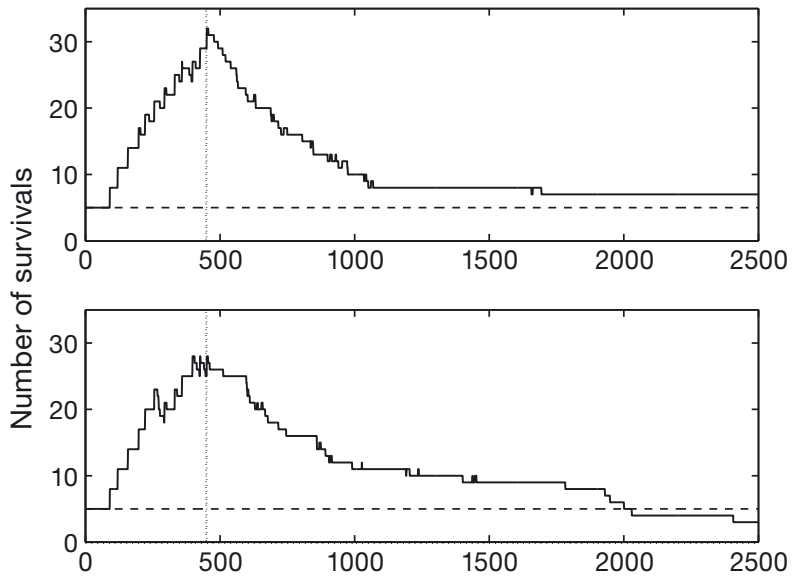
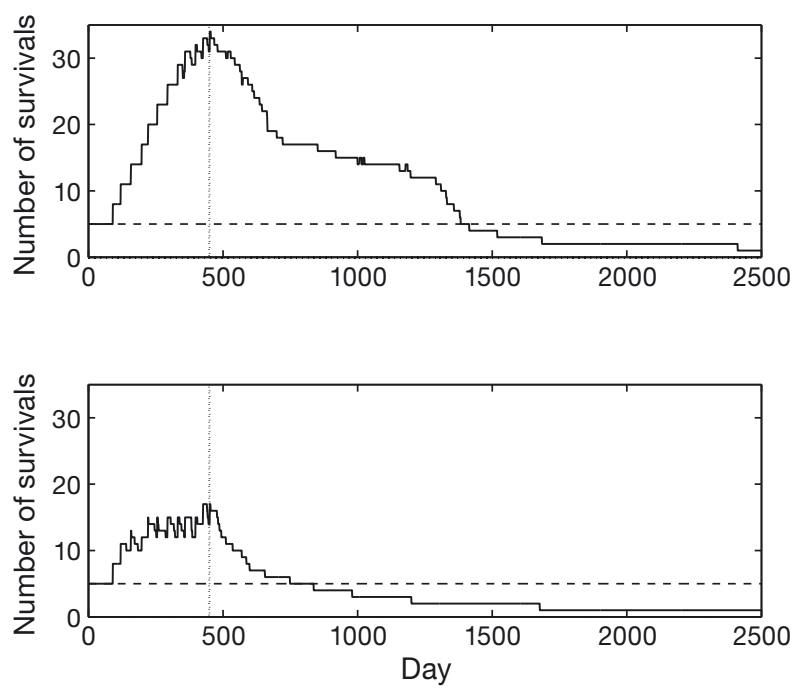

Fig. 6. Phytoplankton species abundance (left panels) and number of survival species (right panels) versus time after 12 intermittent injections of 3 additional species (starting from Day 90 to Day 450, vertical line), depending on whether there is (a) chaos, shown for 2 examples (i) and (ii) with different randomly generated species, (b) oscillations or (c) competitive exclusion. For all cases, there is the same timing of species injections, with the final input indicated by the vertical dotted line. Survival species are defined by the abundance greater than an arbitrary small value of 0.0001 . The horizontal dashed line shows the maximum species number predicted from resource competition theory for an equilibrium state

Hence, none of the species added to the system are fit enough to outcompete the optimal competitor once it is strongly established in the community.

In summary, chaos and oscillations support a comparable number of species, exceeding the number of resources for as long as $2 \mathrm{yr}$ after the last input of new species, while competitive exclusion usually sustains a lower number of species than expected from the resource competition theory (Fig. 8).

\section{DISCUSSION}

Hutchinson (1961) first questioned why so many different phytoplankton species persist, given com- petition theory predicting that at equilibrium the number of species cannot exceed the number of limiting resources. He suggested that this 'paradox of the phytoplankton' and inconsistency with competition theory might be reconciled by the phytoplankton community not being at equilibrium.

There are a variety of explanations as to why an equilibrium state for the phytoplankton community might not be achieved, possibly reflecting a response to the spatial and temporal heterogeneity in the physical environment, or instead an ecological response involving inter-species competition. Phytoplankton species typically have a doubling timescale of 2 to $5 \mathrm{~d}$, and competitive exclusion might be expected to occur over the order of 10 generations, suggesting a time 
(a) Chaos

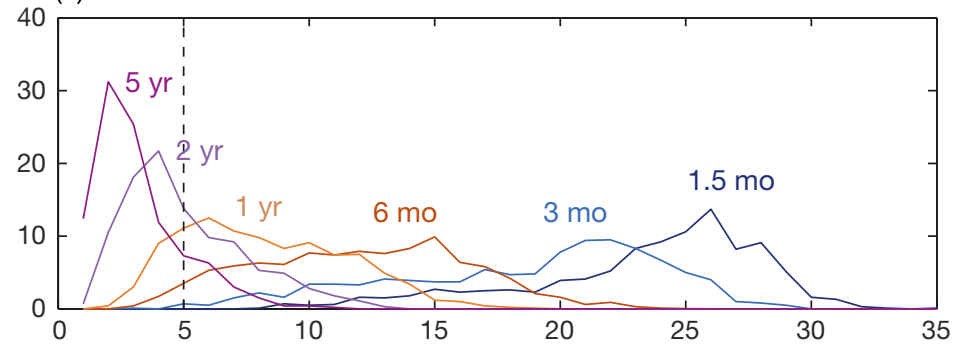

(b) Oscillations

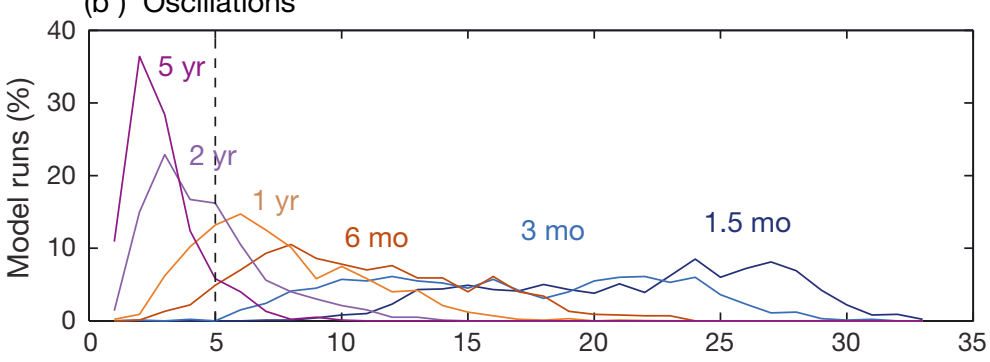

(c) Competitive exclusion

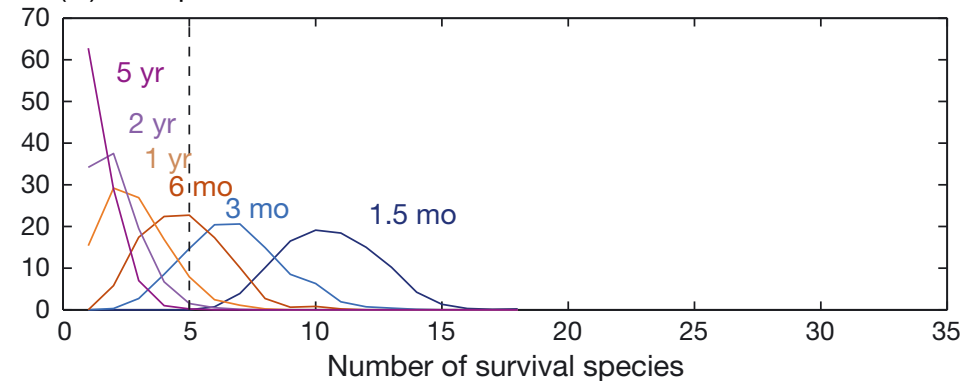

Fig. 7. Number of species sustained at a particular time after the last injection of species, for 1000 model integrations, with each model compilation generated with a different set of random cell traits of injected species. Each set of 1000 runs is induced with different choices of the half-saturation coefficients, $K_{j i}$ for the initial 5 species, which leads to (a) chaos (with default $K_{j i}$ ), (b) 1-period oscillations (with $K_{5,4}=0.37$ ) and (c) competitive exclusion (with $K_{2,4}=0.20$ ). The dashed line indicates the maximum of 5 species surviving on 5 resources predicted for equilibrium by the resource competition theory span for equilibrium to be reached of typically 1 to 2 months (Reynolds 1995). On this timescale, the ocean surface boundary layer is strongly forced by the passage of atmospheric weather systems, modifying the convection and mixing within the surface boundary layer and the light received by phytoplankton. In addition, in coastal seas, the surface boundary layer is modified by the spring-neap changes in the tides. Given this temporal variability in the physical forcing, there are 2 limits leading to relatively low phytoplankton diversity: (1) if there is severe forcing, such as involving a sustained period of no light or nutrient supply followed by an onset of favourable conditions, then the phytoplankton species with the fastest growth rate dominates; and conversely, (2) persistent conditions lead to the optimal competitors flourishing for a stable environment. Hence, the maximum diversity in phytoplankton species is expected between these 2 limits, referred to as the intermediate disturbance hypothesis, which was applied by Connell (1978) for tropical rainforests and coral reefs, discussed for phytoplankton by Padisák (1995) and Reynolds (1995), and used to explain observed changes in the phytoplankton community for a shallow eutrophic lake (Weithoff et al. 2001). Thus, the physical forcing might induce continual temporal and spatial changes in the environment, which the phytoplankton community is continually adjusting to, such that competitive exclusion is not reached.

An alternative view to this physically induced heterogeneity is that there may be

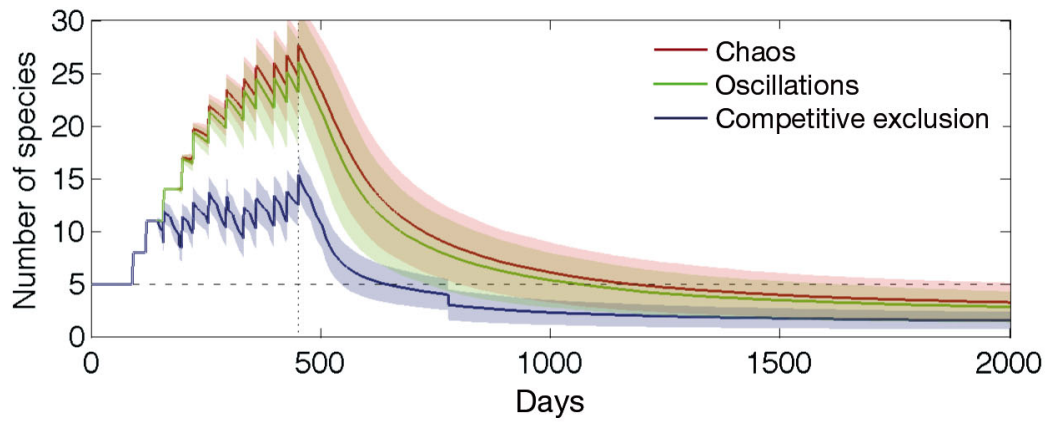

Fig. 8. Mean number of species sustained throughout 1000 model integrations after the last pulse of extra species (indicated by vertical, dotted line). Each set of 1000 runs is induced with different choices of the half-saturation coefficients, $K_{j i}$ for the initial 5 species, which leads to (a) chaos (red line, with default $K_{j i}$ ), (b) 1-period oscillations (green line, with $K_{5,4}=0.37$ and (c) competitive exclusion (blue line, with $K_{2,4}=0.20$ ). SD is indicated by the corresponding shaded regions. The horizontal dashed line specifies the maximum of 5 species coexisting on 5 resources based on the resource competition theory 
more phytoplankton variability due to inter-species competition for resources, as advocated by Huisman \& Weissing $(1999,2001)$. Rather than a single or a few species dominating as in competitive exclusion, the phytoplankton community can continually vary in the form of repeating oscillations or chaotic changes in the abundance of different species.

Whether the model solutions lead to competitive exclusion, oscillations or chaos turns out to be sensitive to the cell physiology and nutrient requirements. Competition between species of similar fitness is most likely to lead to competitive exclusion, with the optimal competitor having the lowest requirement for a resource (Tilman 1977). Including competition between species with variability in cell physiology and nutrient requirement via cell quota does not lead to an optimal competitor emerging, and instead favours oscillatory or chaotic behaviour. The detailed response often turns out to be controlled by the nutrient requirement of the intermediate species compared with that of the other species. In our sets of 1000 model experiments with different ranges in half-saturation coefficient (Table 1), competitive exclusion occurs for $19 \%$ of the integrations if there are large contrasts in half-saturation coefficient, and increases to $47 \%$ of the integrations if there are small contrasts in half-saturation coefficient (Table 1), reflecting the increased chance of identifying an optimal competitor with small contrasts in half saturation. In turn, a combination of oscillations and chaos then occur for at least half of the model integrations.

A particular criticism of whether inter-species competition explains the paradox of the plankton is that chaotic solutions might be an unusual occurrence, as suggested by model experiments initialised with randomly assigned characteristics for the phytoplankton (Schippers et al. 2001). However, this conclusion is challenged by Huisman et al. (2001) who argue that a different response is obtained if additional phytoplankton species are injected at different times and a wider range of physiological choices are made. Our model diagnostics support the view of Huisman \& Weissing $(1999,2001)$ that chaos can emerge in a well-mixed box through inter-species competition for phytoplankton communities. Indeed, a long-term laboratory mesocosm experiment which monitored the plankton community twice a week for $2300 \mathrm{~d}$ revealed chaotic fluctuations in phytoplankton species abundances (Benincà et al. 2008), consistent with a lack of predictability beyond 15 to $30 \mathrm{~d}$.

With respect to how many phytoplankton species are supported when transport and dispersal are included from the wider environment, we find that if there are oscillations or chaotic solutions, then a short-term injection of species leads to a long-term sustenance of more species than resources. In both cases, there is a very similar response with supersaturation in the number of species. In contrast, when there is a competitive exclusion, an additional injection of species only leads to a short-lived excess of species, which quickly die away. Thus, given a random injection of species, both oscillatory and chaotic solutions help sustain more phytoplankton species than resources.

In our model experiments, the emergence of chaos versus oscillations is very sensitive to whether a nutrient feedback is included. When the feedback is strong, chaotic solutions emerge, but when the feedback is weak or absent then the solutions switch to oscillations or competitive exclusion. A choice of strong feedback acting to restore nutrients is appropriate for the way a chemostat operates or for a simple 1-dimensional problem, such as how vertical diffusion acts to supply nutrients down-gradient to the euphotic zone and sustain productivity. However, there is a question as to the extent that the nutrient feedback always holds in the open ocean. The nutrient supply to the euphotic zone is affected by a wide range of physical processes, including convection, entrainment at the base of the mixed layer, and horizontal and vertical transport by the gyre, eddy and basin scale overturning circulations (Williams \& Follows 2003). These processes can either enhance or inhibit biological productivity. For example, winddriven upwelling induces productive surface waters over subpolar gyres, while wind-driven downwelling induces oligotrophic surface waters over subtropical gyres. These physical processes are unlikely to always provide a nutrient feedback to sustain inter-species driven chaos. There may be some regimes, particularly physically isolated cases, when species competition might induce chaos, such as in the deep chlorophyll maximum in oligotrophic gyres during the summer when there is weak mixing (Huisman et al. 2006). Elsewhere, phytoplankton diversity is probably determined by a combination of inter-species competition and the effects of spatial and temporal variations in physical forcing. For example, phytoplankton diversity is enhanced in western boundary currents and gyre boundaries by the combination of transport, lateral mixing and dispersal, as shown by Barton et al. (2010) and Follows et al. (2007).

The sensitivity of our phytoplankton solutions to the coupling between phytoplankton species and the abiotic resource is perhaps analogous to how pre- 
dator-prey cycles and their chaotic solutions are sensitive to the nature of their coupling. For example, coupling of the predator-prey cycles through competition between predators for all prey species leads to predator abundance increasing in phase with the prey, while coupling the cycles of specialist predators leads to the opposite response of prey species declining with increasing predator abundance (Vandermeer 2004, Benincà et al. 2009). The strength of predator-prey interactions also affects whether competitive exclusion, oscillatory or chaotic responses occur (Vandermeer 1993, 2004). Overabundant prey can even destabilize the ecosystem, leading to large amplitude cycles of predator populations (Rosenzweig 1971).

Returning to the question of how the diversity of the phytoplankton community is sustained, as originally posed by Hutchinson (1961), there are 2 apparently contrasting views: the effect of spatial and temporal variability in forcing, and the inter-species competition view. However, both viewpoints involve mechanisms preventing the optimal competitor dominating and leading to an equilibrium state, either achieved via the physical disturbance of the environment or by a transient flourishing of sub-optimal competitors as part of oscillatory and chaotic solutions.

Acknowledgements. K.K. was supported by a UK NERC studentship. We thank A. Barton and M. Begon for insightful discussions, and 3 anonymous referees for their constructive advice and feedback.

\section{LITERATURE CITED}

Armstrong RA, McGehee R (1980) Competitive exclusion. Am Nat 115:151-170

> Barton AD, Dutkiewicz S, Flierl G, Bragg J, Follows MJ (2010) Patterns of diversity in marine phytoplankton. Science 327:1509-1511

> Becks L, Hilker FM, Malchow H, Jürgens K, Arndt H (2005) Experimental demonstration of chaos in a microbial food web. Nature 435:1226-1229

> Benincà E, Huisman J, Heerkloss R, Jöhnk KD and others (2008) Chaos in a long-term experiment with a plankton community. Nature 451:822-825

Benincà E, Jöhnk KD, Heerkloss R, Huisman J (2009) Coupled predator-prey oscillations in a chaotic food web. Ecol Lett 12:1367-1378

Connell JH (1978) Diversity in tropical rain forests and coral reefs - high diversity of trees and corals is maintained only in a non-equilibrium state. Science 199:1302-1310

Droop MR (1973) Some thoughts on nutrient limitation in algae. J Phycol 9:264-272

Follows MJ, Dutkiewicz S, Grant S, Chisholm SW (2007) Emergent biogeography of microbial communities in a model ocean. Science 315:1843-1846

Gottwald GA, Melbourne I (2004) A new test for chaos in deterministic systems. Proc R Soc Lond A 460:603-611

- Gottwald GA, Melbourne I (2009) On the implementation of the 0-1 test for chaos. SIAM J Appl Dyn Syst 8:129-145

> Hardin G (1960) Competitive exclusion principle. Science 131:1292-1297

Hegger R, Kantz H, Schreiber T (1999) Practical implementation of nonlinear time series methods: the TISEAN package. Chaos 9:413-435

Huisman J, Weissing FJ (1999) Biodiversity of plankton by species oscillations and chaos. Nature 402:407-410

Huisman J, Weissing FJ (2001) Biological conditions for oscillations and chaos generated by multispecies competition. Ecology 82:2682-2695

Huisman J, Johansson AM, Folmer EO, Weissing FJ (2001) Towards a solution of the plankton paradox: the importance of physiology and life history. Ecol Lett 4:408-411

Huisman J, Pham Thi NN, Karl DM, Sommeijer B (2006) Reduced mixing generates oscillations and chaos in the oceanic deep chlorophyll maximum. Nature 439:322-325

Hutchinson G (1961) The paradox of the plankton. Am Nat 95:137-145

$>$ Kantz H (1994) A robust method to estimate the maximal Lyapunov exponent of a time series. Phys Lett A 185: $77-87$

Padisák J (1995) Identification of relevant time scales in nonequilibrium community dynamics: conclusions of phytoplankton surveys. NZ J Ecol 18:169-176

Reynolds CS (1995) The intermediate disturbance hypothesis and its applicability to planktonic communities: comments on the views of Padisák and Wilson. NZ J Ecol 19: 219-225

> Richerson P, Armstrong R, Goldman CR (1970) Contemporaneous disequilibrium, a new hypothesis to explain the 'paradox of the plankton'. Proc Natl Acad Sci USA 67: $1710-1714$

Rosenzweig ML (1971) Paradox of enrichment: destabilization of exploitation ecosystems in ecological time. Science 171:385-387

Schippers P, Verschoor A, Vos M (2001) Does 'supersaturated coexistence' resolve the 'paradox of the plankton'? Ecol Lett 4:404-407

> Tilman D (1977) Resource competition between planktonic algae-experimental and theoretical approach. Ecology $58: 338-348$

Tilman D (1980) Resources: a graphical-mechanistic approach to competition and predation. Am Nat 116: 362-393

Tilman D, Kilham SS, Kilham P (1982) Phytoplankton community ecology - the role of limiting nutrients. Annu Rev Ecol Syst 13:349-372

- Vandermeer J (1993) Loose coupling of predator-prey cycles: entrainment, chaos, and intermittency in the classic MacArthur consumer-resource equations. Am Nat 141:687-716

> Vandermeer J (2004) Coupled oscillations in food webs: balancing competition and mutualism in simple ecological models. Am Nat 163:857-867

Weithoff G, Walz N, Gaedke U (2001) The intermediate disturbance hypothesis - species diversity or functional diversity? J Plant Res 23:1147-1155

Williams RG, Follows MJ (2003) Physical transport of nutrients and the maintenance of biological production. In: Fasham M (ed) Ocean biogeochemistry: the role of the ocean carbon cycle in global change. Springer-Verlag, Berlin, p 19-51 